\title{
GRAPH SPACES AND $\perp$-FREE BOOLEAN ALGEBRAS
}

\author{
LUTZ HEINDORF
}

(Communicated by Franklin D. Tall)

\begin{abstract}
Let $X$ denote an arbitrary second-countable, compact, zero-dimensional space. Our main result says that $X$ is a graph space, i.e., homeomorphic to the space of all complete subgraphs of a suitable graph. We first characterize graph spaces in terms of the Boolean algebras of their clopen subsets. Then it is proved that each countable Boolean algebra has the corresponding property.

As a corollary we obtain that $X$ is homeomorphic to the underlying space of a subalgebra of $\langle 2 ; F\rangle^{\omega}$, where 2 is the discrete two-point space and $F$ any set of finitary operations on 2 such that neither the negation nor the ternary sum $x \oplus y \oplus z$ (addition modulo 2) belongs to the clone generated by $F$.
\end{abstract}

\section{INTRODUCTION}

The concept of a graph space has been introduced by M. G. Bell who used it in [1] as a tool to construct examples of peculiar spaces.

For us a graph $\mathscr{G}$ will be a pair $\langle G ; R\rangle$ where $G$ is a set and $R$ a reflexive and symmetric binary relation on $G$. Given such a graph, we denote by $C(\mathscr{G})$ the subspace of $2^{G}$ consisting of all $\varphi: G \rightarrow 2$ such that

$$
\varphi\left(g_{1}\right)=\varphi\left(g_{2}\right)=1 \text { implies }\left(g_{1}, g_{2}\right) \in R .
$$

Here and below $\mathbf{2}=\{0,1\}$ is considered with the discrete topology and $\mathbf{2}^{G}$ with the product topology. Condition $(*)$ says that $\varphi$ is the characteristic function of a (possibly empty) complete subgraph of $\mathscr{G}$. This motivates the letter $C$ in $C(\mathscr{G})$.

It is easy to see that $C(\mathscr{G})$ is always closed in $2^{G}$, so it is a compact and zero-dimensional space. For short, in what follows we simply say space instead of compact zero-dimensional space (no others occur in this paper). Let us also agree that $X$ always denotes a space. We call $X$ a graph space iff it is homeomorphic to $C(\mathscr{G})$ for some graph $\mathscr{G}$.

To each space $X$ Stone duality attaches the Boolean algebra $\operatorname{Clop}(X)$ of its clopen subsets under the set-theoretic operations. To understand the proofs below, the reader is supposed to have some experience with Boolean algebras. In contrast to what has now become fashionable, e.g., in [3], we still use $\vee$ and $\wedge$ to denote join and meet in an abstract Boolean algebra. 0 and 1 are the universal bounds. For a finite subset $F=\left\{f_{1}, \ldots, f_{n}\right\}$ of some Boolean algebra we

Received by the editors July 22, 1992.

1991 Mathematics Subject Classification. Primary 06E15, 54D80, 54H12. 
alternatively use $\bigvee F$ and $\bigvee_{i=1}^{n} f_{i}$ to denote its least upper bound (analogously with $\Lambda$ ). The difference between the unary operation of complementation and the binary operation of relative complementation will be important in some places. We stress that difference by using $\neg$ for the former and - for the latter, i.e., $\neg a=1-a$ and $a-b=a \wedge \neg b$. In Clop the operations $\wedge, \vee$, and - correspond to $\cap, \cup$, and $\backslash$, respectively.

It will be convenient to have a special symbol to denote disjointness: $a \perp b$ means $a \wedge b=0$.

Let $A$ and $B$ denote Boolean algebras and $D$ a subset of $A$. We call a mapping $\varphi: D \rightarrow B \perp$-preserving iff $d_{1} \perp d_{2}$ implies $\varphi\left(d_{1}\right) \perp \varphi\left(d_{2}\right)$ for all $d_{1}, d_{2} \in D$. It is clear that every restriction to $D$ of a homomorphism $A \rightarrow B$ is $\perp$-preserving. We shall be interested in situations where the opposite is true.

We say that $A$ is $\perp$-free over $D$ iff each $\perp$-preserving mapping $\varphi: D \rightarrow$ $B$ into an arbitrary Boolean algebra $B$ extends to a unique homomorphism $\bar{\varphi}: A \rightarrow B$. Notice that the uniqueness requirement simply means that $D$ generates $A . A$ is called $\perp$-free iff it is $\perp$-free over some subset $D$.

Having introduced these notions, we can give a preview of the paper. In the next section we prove that $X$ is a graph space iff $\operatorname{Clop}(X)$ is $\perp$-free. Our main result will be proved in $\S 2$ : all second-countable spaces are graph spaces, or, equivalently, all countable Boolean algebras are $\perp$-free. In the final section we address a seemingly unrelated problem. Under which conditions on the set $F$ of finitary operations on the set 2 is it possible to realize each second-countable space as a subalgebra of $\langle 2 ; F\rangle^{\omega}$ ? The answer comes as a corollary of clone theory and our main result: The clone generated by $F$ must neither contain the negation $x \mapsto \neg x$ nor the affine sum $x, y, z \mapsto x \oplus y \oplus z$ (addition modulo 2 ). Contrary to $\S \S 1$ and 2 that are more or less self-contained, the third section relies on Post's classification of all clones on a two element set, without giving any explanation about it.

\section{Algebra Versus tOPOlOGY}

In this section we prove that $X$ is a graph space iff $\operatorname{Clop}(X)$ is $\perp$-free. One direction is simple. Indeed, assume that $\operatorname{Clop}(X)$ is $\perp$-free over its subset $D$. A quick look at the definition shows that we can assume $0 \notin D$. Put $R=\left\{\left(d_{1}, d_{2}\right) \in D^{2}: d_{1} \wedge d_{2} \neq 0\right\}$ and $\mathscr{G}=\langle D ; R\rangle$. Define $f: X \rightarrow \mathbf{2}^{D}$ by setting $f(x)(d)=1$ iff $x \in d$. It is obvious that each $f(x)$ satisfies (*). So, $f$ maps $X$ into $C(\mathscr{G})$. We check that $f$ is a homeomorphism. Continuity is easy. Injectivity follows from the fact that $D$ generates $\operatorname{Clop}(X)$. To prove surjectivity, consider any $\varphi \in C(\mathscr{G})$. Condition $(*)$ in our context means that $\varphi: D \rightarrow\langle 2 ; \wedge, \vee, \neg, 0,1\rangle$ preserves $\perp$. Therefore, by $\perp$-freedom, $\varphi$ extends to a homomorphism $\bar{\varphi}: \operatorname{Clop}(X) \rightarrow\langle 2 ; \wedge, \vee, \neg, 0,1\rangle$. By Stone duality, there exists a point $x \in X$ such that $\bar{\varphi}(a)=1$ iff $x \in a$. It follows that $\varphi=\bar{\varphi} \mid D=f(x)$.

A direct proof of the other direction is also not difficult. In view of later applications, we prefer to prove it in three steps, inserting two more assertions. To state these, we need some more notation.

Definition 1.1. We call a subset $D$ of a Boolean algebra $\perp$-independent iff for all nonempty finite subsets $F$ and $G$ of $D$ the following conditions hold:

$(\perp 1) \bigvee F \neq 1$ 
( $\perp$ 2) If $\wedge F=0$, then $f_{1} \wedge f_{2}=0$ for some $f_{1}, f_{2} \in F$.

( $\perp 3)$ If $0 \neq \wedge F \leq \bigvee G$, then $F \cap G \neq \varnothing$.

Furthermore we need a name for a particular ternary operation on the set 2 . In terms of the Boolean operations it is defined by

$$
m(x, y, z)=(x \wedge y) \vee(x \wedge z) \vee(y \wedge z) .
$$

Algebraists call $m$ the median operation, whereas topologists like the word mixer. It yields the repeated value among $x, y, z$. Recall that - denotes the binary relative complement operation. We consider $\langle 2 ; m,-\rangle$ as a topological algebra and likewise its countable power $\langle 2 ; m,-\rangle^{\omega}$. The former is discrete, whereas the latter carries the product topology. We need the following assertion about these algebras.

Lemma 1.2. For all $n, k$ the $(n k+1)$-ary operation

$$
x \wedge\left[y_{11} \vee \cdots \vee y_{1 n}\right] \wedge \cdots \wedge\left[y_{k 1} \vee \cdots \vee y_{k n}\right]
$$

can be expressed in terms of $m$ and - .

Proof. For the initiated it is sufficient to observe that the function in question belongs to the clone $F_{8}^{2}$ (in Post's notation), which is generated by $m$ and - . The others have to verify the following equations, which combined allow us to write down the necessary term.

(1) $x \wedge y=m(x, y, x-x)$.

(2) $x \wedge\left(y_{1} \vee y_{2}\right)=m\left(x, y_{1}, y_{2}-y_{1}\right)$.

(3) $x \wedge\left(y_{1} \vee \cdots \vee y_{n+1}\right)=x \wedge\left\{\left[x \wedge\left(y_{1} \vee \cdots \vee y_{n}\right)\right] \vee\left[\left(y_{n+1}-y_{1}\right) \wedge \cdots \wedge\left(y_{n+1}-y_{n}\right)\right]\right\}$.

(4) $x \wedge\left[y_{11} \vee \cdots \vee y_{1 n}\right] \wedge \cdots \wedge\left[y_{k 1} \vee \cdots \vee y_{k n}\right]=\left[x \wedge\left(y_{11} \vee \cdots \vee y_{1 n}\right)\right] \wedge \cdots \wedge$ $\left[x \wedge\left(y_{k 1} \vee \cdots \vee y_{k n}\right)\right]$.

Theorem 1.3. For each compact and zero-dimensional space $X$ the following assertions are equivalent.

(1) $X$ is a graph space.

(2) $X$ is homeomorphic to (the underlying space of) a subalgebra of some power $\langle 2 ; m,-\rangle^{I}$.

(3) $\operatorname{Clop}(X)$ is generated by some $\perp$-independent subset.

(4) $\operatorname{Clop}(X)$ is $\perp$-free.

Proof. An easy calculation shows that $C(\mathscr{G})$ is a subalgebra of $\langle 2 ; m,-\rangle^{G}$. This proves $(1) \Rightarrow(2)$. The implication $(3) \Rightarrow(4)$ is a straightforward application of Sikorski's Extension Criterion (Theorem 5.5 of [3]) and (4) $\Rightarrow(1)$ has been proved at the beginning of this section.

So we are left with $(2) \Rightarrow(3)$. Assume that $X \subseteq 2^{I}$ is closed under $m$ and - . It will be convenient to identify the clopen subsets of $X$ with their characteristic functions. $\operatorname{Clop}(X)$ then becomes the subalgebra of $\langle 2 ; \wedge, \vee, \neg, 0,1\rangle^{X}$ consisting of all continuous mappings. Let $D \subseteq \operatorname{Clop}(X)$ consist of those continuous mappings $d: X \rightarrow 2$ that preserve the operations $m$ and -. First of all, $D$ separates the points of $X$. For, the restrictions to $X$ of the projections $2^{I} \rightarrow 2$ do, and these belong to $D$. It follows that $D$ generates $\operatorname{Clop}(X)$ as a Boolean algebra. Next we consider two subsets $F=\left\{f_{1}, \ldots, f_{n}\right\}$ and $G=\left\{g_{1}, \ldots, g_{m}\right\}$ of $D$ and verify the three clauses in the definition of $\perp$ independence. 
$(\perp 1)$ Take any $x \in X$ and put $y=x-x$. Then $d(y)=0$ for all $d \in D$. In particular, $\bigvee_{i=1}^{n} f_{i}(y)=0$, so $\bigvee F \neq 1$.

$(\perp 2)$ By induction on $n$ we prove that if $f_{i} \wedge f_{j} \neq 0$ for all $i, j \leq n$, then $\bigwedge_{i=1}^{n} f_{i} \neq 0$. This is obvious for $n=2$. Suppose it is true for $n$. Then we find $x_{1}, x_{2}, x_{3} \in X$ such that:

$$
\begin{aligned}
& f_{2}\left(x_{1}\right) \wedge f_{3}\left(x_{1}\right) \wedge \bigwedge_{i=4}^{n+1} f_{i}\left(x_{1}\right)=1 \\
& f_{1}\left(x_{2}\right) \wedge \quad f_{3}\left(x_{2}\right) \wedge \wedge_{i=4}^{n+1} f_{i}\left(x_{2}\right)=1 \\
& f_{1}\left(x_{3}\right) \wedge f_{2}\left(x_{3}\right) \quad \wedge \wedge_{i=4}^{n+1} f_{i}\left(x_{3}\right)=1
\end{aligned}
$$

For $y=m\left(x_{1}, x_{2}, x_{3}\right)$, it follows that $\bigwedge_{i=1}^{n+1} f_{i}(y)=1$.

( $\perp 3)$ Assume that $\bigwedge_{i=1}^{n} f_{i}(x) \leq \bigvee_{j=1}^{m} g_{j}(x)$ for all $x \in X$, and that there is some $x_{0}$ such that $\bigwedge_{i=1}^{n} f_{i}\left(x_{0}\right)=1$.

Claim. There is a pair $i, j$ of indices such that $f_{i}(x) \leq g_{j}(x)$ for all $x \in X$.

If that were not the case, we could take $y_{i j} \in X$ such that $f_{i}\left(y_{i j}\right)=1$ and $g_{j}\left(y_{i j}\right)=0$. By the lemma, there is a point $z \in X$ such that

$$
d(z)=d\left(x_{0}\right) \wedge\left[d\left(y_{11}\right) \vee \cdots \vee d\left(y_{n 1}\right)\right] \wedge \cdots \wedge\left[d\left(y_{1 m}\right) \vee \cdots \vee d\left(y_{n m}\right)\right]
$$

for all $d \in D$. Substituting $d=f_{i}$ we get $f_{i}(z)=1$ for all $i$. Substituting $d=g_{j}$ we get $g_{j}(z)=0$ for all $j$. This contradicts $\wedge f_{i} \leq \bigvee g_{j}$ and proves the claim.

It remains to see that, actually, $f_{i}(x)=g_{j}(x)$ for all $x$. But, otherwise, we would find some $y_{0}$ such that $f_{i}\left(y_{0}\right)=0$ and $g_{j}\left(y_{0}\right)=1$. This would imply $f_{i}\left(x_{0}-y_{0}\right)=1 \not \leq 0=g_{j}\left(x_{0}-y_{0}\right)$.

\section{The MAIN RESULT}

Let $\mathscr{G}$ be a graph and consider the mapping $\zeta: G \rightarrow 2$ which sends each $g \in G$ to 0 . Then $\zeta \in C(\mathscr{G})$ and it is not hard to check that $\chi(\zeta, C(\mathscr{G}))=$ $w(\mathscr{G})=|G|$. As there are spaces without a point whose character equals weight, not all spaces are graph spaces. On the other hand, we have the following positive result.

Theorem 2.1. Every second-countable, compact, zero-dimensional space is a graph space.

In view of Theorem 1.3 it is sufficient to show that every countable Boolean algebra is generated by some $\perp$-independent set. For the rest of this section we fix a countable Boolean algebra $A$ and its enumeration $\left(a_{n}\right)_{n<\omega}$. The proof will consist in the inductive construction of an increasing sequence $\left(D_{n}\right)_{n<\omega}$ of $\perp$ independent finite subsets of $A$ such that $a_{n} \in\left\langle D_{n+1}\right\rangle$. Here $\langle D\rangle$ denotes the subalgebra of $A$ generated by $D$. It is clear that $\bigcup_{n<\omega} D_{n}$ will be the desired $\perp$-independent set generating $A$.

In the inductive step we are confronted with the following difficulty. If $a_{n} \notin$ $\left\langle D_{n}\right\rangle$, then $a_{n}$ splits some atom, $b$ say, of $\left\langle D_{n}\right\rangle$, i.e., $b \wedge a_{n} \neq 0$ and $b-a_{n} \neq 0$. Thus $D_{n+1}$ must include some $e$ which also splits $b$. But, for $D_{n} \cup\{e\}$ to remain $\perp$-independent it may be necessary that $e$ splits further atoms of $\left\langle D_{n}\right\rangle$. These further atoms depend on the position of $b$ and it will be our first concern to clarify this dependence. Having done that we secure by induction that all the necessary atoms of $\left\langle D_{n}\right\rangle$ can indeed be split. 
From now on we let $D$ denote a finite subset of $A$. We define a mapping $P$ from $A t\langle D\rangle$, the set of all atoms of $\langle D\rangle$, into the power-set of $D$ by setting

$$
P(a)=\{d \in D: a \leq d\} .
$$

Notice that $P$ is injective. It follows that by setting

$$
a \preceq b \quad \text { iff } P(b) \subseteq P(a)
$$

we define a (reflexive) partial order on $A t\langle D\rangle$.

Lemma 2.2. Suppose that $a \in A t\langle D\rangle$ and $e \in A$ are such that for all $b \in A t\langle D\rangle$

$$
e \wedge b \neq 0 \text { iff } e \text { splits } b \text { iff } a \preceq b .
$$

If $D$ is $\perp$-independent, then $D \cup\{e\}$ is also $\perp$-independent.

Proof. Let us denote $\{b \in A t\langle D\rangle: a \preceq b\}$ by $B$ and $A t\langle D\rangle \backslash B$ by $C$. Then $e \leq \bigvee B$ and $e \wedge \bigvee C=0$. Immediately from the assumption about $a$ and $e$ we get

Claim 1. There is no element of $\langle D\rangle$ below e, except 0 .

The next observation will be used twice.

Claim 2. For all $d \in D$, if $d \wedge a=0$, then $d \wedge e=0$.

Indeed, as $a$ is an atom of $\langle D\rangle, d \wedge a=0$ is equivalent to $d \notin P(a)$. But $P(a) \supseteq P(b)$ for all $b \in B$. Hence, $d \wedge e \leq d \wedge \bigvee B=0$.

Suppressing trivial cases we now verify all three clauses of Definition 1.1.

$(\perp 1)$ Put $u=1-\bigvee D$. Then $u \neq 0$, by $\perp$-independence of $D$, hence $u \in A t\langle D\rangle$. From $P(u)=\varnothing \subseteq P(a)$, i.e., $a \preceq u$, we get $0<e \wedge u<u$, by assumption. Hence $0<u-e=1-\bigvee(D \cup\{e\})$.

$(\perp 2)$ Let $F \subset D$ be such that $\wedge F \wedge e=0$. From $a \leq \wedge F$, we would get $0<a \wedge e \leq \wedge F \wedge e$, a contradiction. Hence, $f \wedge a=0$ for some $f \in F$. But then $f \wedge e=0$, by Claim 2 .

( $\perp$ 3) splits into two nontrivial subcases, namely (a) $0<\wedge F \leq \bigvee G \vee e$ and (b) $0<\wedge F \wedge e \leq \bigvee G$.

In case (a) we have $\wedge F-\bigvee G \leq e$. By Claim $1, \wedge F-\bigvee G=0$, hence $F \cap G \neq \varnothing$, by $\perp$-independence of $D$.

In case (b) we claim that

$$
\bigwedge F \leq \bigvee G \vee \bigvee\{d \in D: d \wedge a=0\}
$$

To see this, consider any $c \in A t\langle D\rangle$ below $\wedge F$. Either $c \wedge e \neq 0$, so $0<$ $c \wedge(\wedge F \wedge e) \leq c \wedge \bigvee G$ and hence $c \leq \bigvee G$, or $c \wedge e=0$. Then $a \preceq c$ is not true, by assumption, and there exists some $d \in D$ such that $d \geq c$ and $d \wedge a=0$.

From $(+)$ and the $\perp$-independence of $D$ we either get $F \cap G \neq \varnothing$, as desired, or, $f \wedge a=0$ for some $f \in F$. By Claim 2, the latter would imply $0=f \wedge e \geq \wedge F \wedge e$, a contradiction.

Next we describe the partial order on $A t\langle D \cup\{e\}\rangle$. We use $\preceq_{e}$ to distinguish it from $\preceq$ on $A t\langle D\rangle$. Analogously, for $f \in A t\langle D \cup\{e\}\rangle$ we put $P_{e}(f)=\{g \in$ $D \cup\{e\}: f \leq g\}$. Let us keep the notation $B=\{b \in A t\langle D\rangle: a \preceq b\}$ and $C=A t\langle D\rangle \backslash B$ from the previous proof. 
Lemma 2.3. Under the assumptions of Lemma 2.2 the following assertions hold.

(1) $A t\langle D \cup\{e\}\rangle=\{b \wedge e: b \in B\} \cup\{b-e: b \in B\} \cup C$.

(2) The mapping sending $b \in B$ to $b-e$ and $c \in C$ to itself is order preserving ( $\preceq$ versus $\preceq_{e}$ ).

(3) The mapping sending $b \in B$ to $b \wedge e$ is order preserving.

(4) For $b \in B$ and $c \in C$, the elements $b \wedge e$ and $c$ are incomparable with respect to $\preceq_{e}$.

(5) For $b_{1}, b_{2} \in B, b_{1} \wedge e \preceq_{e} b_{2}-e$ iff $b_{1} \preceq b_{2}$.

(6) For $b_{1}, b_{2} \in B$, not $b_{1}-e \preceq_{e} b_{2} \wedge e$.

Proof. The proof is a straightforward verification. (1) is obvious. The other assertions rely on the following three identities that are in turn easily checked. For all $b \in B$ and $c \in C$,

$$
\begin{aligned}
P_{e}(b \wedge e) & =P(b) \cup\{e\}, \\
P_{e}(b-e) & =P(b), \\
P_{e}(c) & =P(c) .
\end{aligned}
$$

As an example we check (4): $P_{e}(b \wedge e) \subseteq P_{e}(c)$ is impossible, because $e$ belongs to the left-hand side. $P_{e}(c) \subseteq P_{e}(b \wedge e)$ would imply $P(c) \subseteq P(b)$, i.e., $b \preceq c$ which together with $a \preceq b$ would yield $a \preceq c$, i.e., $c \in B$, a contradiction.

The condition that the necessary atoms of $\langle D\rangle$ can be split will be stated in terms of rank and degree. In order to make the argument easier for topologists, we express these notions in topological terms, assuming $A=\operatorname{Clop}(X)$ for some space $X$.

Let $\operatorname{CBR}(x)$ denote the Cantor-Bendixson-Rank of the point $x \in X$. To be definite: isolated points have rank one, points in the perfect kernel have rank $\infty$. Each $a \in A$ is a compact subset of $X$ which may or may not be scattered. We put $\operatorname{rk}(a)=\max \{\operatorname{CBR}(x): x \in a\}$ and $\operatorname{deg}(a)=\mid\{x \in a: \operatorname{CBR}(x)=$ $\operatorname{rk}(a)\} \mid$, if $a$ is scattered, and $\operatorname{rk}(a)=\infty$ and $\operatorname{deg}(a)=1$, otherwise.

Notice that deg is always finite. As we consider $\varnothing$ as scattered, we have $\operatorname{rk}(\varnothing)=\operatorname{deg}(\varnothing)=0$. In the argument below we use some of the easy properties of rank and degree, such as: if $\operatorname{rk}(a)=\infty$, then there is some $b \leq a$ such that $\operatorname{rk}(b)=\operatorname{rk}(a-b)=\infty$.

It will be convenient to call a finite subset $D \subseteq A$ extendable if it is $\perp$ independent and for all $a, b \in A t\langle D\rangle$

(e1) $\operatorname{deg}(a)=1$,

(e2) $a \preceq b$ implies $\operatorname{rk}(a) \leq \operatorname{rk}(b)$.

The following is the justification for the word 'extendable' and the key for the inductive construction.

Lemma 2.4. For each extendable $D \subseteq A$ and for every $f \in A$ there exists an extendable $D^{\prime} \subseteq A$ such that $D \subseteq D^{\prime}$ and $f \in\left\langle D^{\prime}\right\rangle$.

Proof. We proceed by induction on

$$
\operatorname{size}(f, D)=\sum\{\operatorname{deg}(a \wedge f)+\operatorname{deg}(a-f): f \text { splits } a \in A t\langle D\rangle\} .
$$

If $\operatorname{size}(f, D)$ is zero, then $f$ does not split any atom of $\langle D\rangle$. It follows that $f$ belongs to $\langle D\rangle$ and $D^{\prime}=D$ does the job.

Assume then that $\operatorname{size}(f, D)>0$ and take any $a \in A t\langle D\rangle$ that is split by $f$. As in the previous proof we put $B=\{b \in A t\langle D\rangle: a \preceq b\}$ and $C=A t\langle D\rangle \backslash B$. Let $\left\{b_{0}, \ldots, b_{n}\right\}$ be an enumeration of $B$ such that $b_{0}=a$. 
We are going to construct elements $e_{i}$ such that $0<e_{i}<b_{i}$. After that we put $e=e_{0} \vee \cdots \vee e_{n}$ and $D^{\prime}=D \cup\{e\}$. By Lemma 2.2, $D^{\prime}$ will then be $\perp-$ independent. The choice of the $e_{i}$ has to further guarantee conditions $(\mathrm{e} 1)$ and (e2) and that $\operatorname{size}\left(f, D^{\prime}\right)<\operatorname{size}(f, D)$. The final choice of the $e_{i}$ will depend on whether $i=0$ or $i>0$. We first discuss the features that are common for all $i$. In all cases $b_{i}^{\prime}$ will be one of $b_{i} \wedge f$ or $b_{i}-f$, and $0<e_{i} \leq b_{i}^{\prime}$ is chosen in such a way that

$$
\operatorname{deg}\left(e_{i}\right)=1
$$

and

$(i, * *)$

$$
\operatorname{rk}\left(e_{i}\right) \leq \operatorname{rk}\left(b_{i}-e_{i}\right) .
$$

These choices have the following:consequences:

$$
\left\{b_{i} \wedge f, b_{i}-f\right\}=\left\{b_{i}^{\prime}, b_{i}-b_{i}^{\prime}\right\}
$$

which is obvious,

$$
\left\{\left(b_{i}-e_{i}\right) \wedge f,\left(b_{i}-e_{i}\right)-f\right\}=\left\{b_{i}^{\prime}-e_{i}, b_{i}-b_{i}^{\prime}\right\},
$$

which follows from $(i, 0)$ and $e_{i} \leq b_{i}^{\prime}$,

$$
\operatorname{deg}\left(b_{i}-e_{i}\right)=1 \text {, }
$$

which follows from $(i, * *)$ and $\operatorname{deg}\left(b_{i}\right)=1$ (i.e., (e1) for $D$ ), and

$$
\operatorname{rk}\left(b_{i}-e_{i}\right)=\operatorname{rk}\left(b_{i}\right)
$$

by $(i, * *)$.

Choice of $e_{0}$. Recall that $b_{0}=a$. By assumption, $b_{0} \wedge f$ and $b_{0}-f$ are both nonzero. It follows that we can take $b_{0}^{\prime}$ among them such that $0<$ $\operatorname{rk}\left(b_{0}^{\prime}\right) \leq \operatorname{rk}\left(b_{0}-b_{0}^{\prime}\right)=\operatorname{rk}\left(b_{0}\right)$. Then take $0<e_{0} \leq b_{0}^{\prime}$ such that $\operatorname{deg}\left(e_{0}\right)=1$, and $\operatorname{deg}\left(b_{0}^{\prime}-e_{0}\right)<\operatorname{deg}\left(b_{0}^{\prime}\right)$. Condition $(0, *)$ is explicitly satisfied and $(0, * *)$ follows because $\operatorname{rk}\left(e_{0}\right) \leq \operatorname{rk}\left(b_{0}^{\prime}\right) \leq \operatorname{rk}\left(b_{0}-b_{0}^{\prime}\right) \leq \operatorname{rk}\left(b_{0}-e_{0}\right)$. From $(0,0)$, $(0,1)$, and $\operatorname{deg}\left(b_{0}^{\prime}-e_{0}\right)<\operatorname{deg}\left(b_{0}^{\prime}\right)$ we conclude that

$$
(0,4) \quad \operatorname{deg}\left(\left(b_{0}-e_{0}\right) \wedge f\right)+\operatorname{deg}\left(\left(b_{0}-e_{0}\right)-f\right)<\operatorname{deg}\left(b_{0} \wedge f\right)+\operatorname{deg}\left(b_{0}-f\right) \text {. }
$$

Choice of $e_{i}$ for $i>0$. This time we let $b_{i}^{\prime}$ be such that $\operatorname{rk}\left(b_{i}-b_{i}^{\prime}\right) \leq$ $\operatorname{rk}\left(b_{i}^{\prime}\right)=\operatorname{rk}\left(b_{i}\right)$. If $\operatorname{rk}\left(b_{i}^{\prime}\right)=\infty$, then we take $e_{i} \leq b_{i}^{\prime}$ such that $\operatorname{rk}\left(e_{i}\right)=$ $\operatorname{rk}\left(b_{i}^{\prime}-e_{i}\right)=\infty$. Conditions $(i, *)$ and $(i, * *)$ follow automatically. Suppose now that $\operatorname{rk}\left(b_{i}^{\prime}\right)<\infty$. By $(\mathrm{e} 2)$ for $D$ and $b_{0} \preceq b_{i}$ we have that $\operatorname{rk}\left(b_{0}\right) \leq$ $\operatorname{rk}\left(b_{i}\right)=\operatorname{rk}\left(b_{i}^{\prime}\right)<\infty$. As $\operatorname{deg}\left(b_{0}\right)=1$ and $\operatorname{rk}\left(e_{0}\right) \leq \operatorname{rk}\left(b_{0}-e_{0}\right)$, we must have that $\operatorname{rk}\left(e_{0}\right)<\operatorname{rk}\left(b_{0}\right) \leq \operatorname{rk}\left(b_{i}^{\prime}\right)$. It follows that we can take $e_{i} \leq b_{i}^{\prime}$ such that $\operatorname{deg}\left(e_{i}\right)=1$ and $\operatorname{rk}\left(e_{i}\right)=\operatorname{rk}\left(e_{0}\right)$. Again $(i, *)$ and $(i, * *)$ are obviously satisfied.

From $\operatorname{rk}\left(e_{i}\right)<\operatorname{rk}\left(b_{i}^{\prime}\right)$ it also follows that $\operatorname{deg}\left(b_{i}^{\prime}-e_{i}\right)=\operatorname{deg}\left(b_{i}^{\prime}\right)$, which together with $(i, 0)$ and $(i, 1)$ yields

$$
(i, 4) \quad \operatorname{deg}\left(\left(b_{i}-e_{i}\right) \wedge f\right)+\operatorname{deg}\left(\left(b_{i}-e_{i}\right)-f\right)=\operatorname{deg}\left(b_{i} \wedge f\right)+\operatorname{deg}\left(b_{i}-f\right) .
$$

The construction is complete. As mentioned above, by setting $e=e_{0} \vee \cdots \vee e_{n}$ and $D^{\prime}=D \cup\{e\}$, we get a $\perp$-independent set.

The atoms of $\left\langle D^{\prime}\right\rangle$ are $e \wedge b_{i}=e_{i}$ and $b_{i}-e=b_{i}-e_{i}$ for $b_{i} \in B$ together with the untouched $c \in C$. By $(i, *)$ and $(i, 2)$ they all have degree one, 
which yields (e1). To check that $x \preceq_{e} y$ implies $\operatorname{rk}(x) \leq \operatorname{rk}(y)$, one has to use Lemma 2.3 and conditions $(i, 3)$ and $(i, * *)$. The only nonobvious case may be $e_{i} \preceq_{e} e_{j}$. Both ranks are either equal to $\operatorname{rk}\left(e_{0}\right)$ or $\infty$. But $\operatorname{rk}\left(e_{i}\right)=\infty$ can only occur if $\operatorname{rk}\left(b_{i}\right)=\infty$. In this case we have $\operatorname{rk}\left(b_{j}\right)=\infty$, since $b_{i} \preceq b_{j}$ and $D$ is extendable. Hence $\mathrm{rk}\left(e_{j}\right)=\infty$, by construction.

We have proved that $D^{\prime}$ is extendable. To evaluate $\operatorname{size}\left(f, D^{\prime}\right)$ we remark that $f$ does not split any $e_{i}$ (because $f$ does not split $b_{i}^{\prime} \geq e_{i}$ ). Moreover, if $f$ splits $b_{i}-e_{i}$, then $f$ splits $b_{i}$. By $(i, 4)$ we get that the term

$$
\operatorname{deg}\left(\left(b_{i}-e_{i}\right) \wedge f\right)+\operatorname{deg}\left(\left(b_{i}-e_{i}\right)-f\right)
$$

in the sum making up $\operatorname{size}\left(f, D^{\prime}\right)$ has not changed for $i>0$ and strictly fallen for $i=0$. It follows that the size has fallen, which ends the proof of the lemma.

Now it is clear how to prove Theorem 2.1. For some $m>0$ we take pairwise disjoint elements $d_{1}, \ldots, d_{m+1} \in A$ of degree 1 , such that $d_{1} \vee \cdots \vee d_{m+1}=1$. Then $D_{0}=\left\{d_{1}, \ldots, d_{m}\right\}$ is easily seen to be $\perp$-independent and extendable. Now Lemma 2.4 allows us to carry out the inductive construction described after the statement of the theorem.

\section{WHICH SPACES ADMIT WHICH OPERATIONS}

Let $F$ be any set of finitary operations on 2 . We consider $\langle 2 ; F\rangle$ as a discrete topological algebra. Its infinite powers $\langle 2 ; F\rangle^{I}$ are topological algebras whose underlying spaces are Cantor cubes of weight $|I|$. It is well known that every space $X$ can be embedded into a Cantor cube. In this section we investigate whether the embedding can be chosen in such a way that $X$ becomes a subalgebra of $\langle 2 ; F\rangle^{I}$. Let us say that $X$ admits $F$ if such an embedding is possible. For example, in the previous section we proved that all secondcountable spaces admit $F=\{m,-\}$. On the other hand, there are spaces that do not admit any nontrivial binary operation. For, if $X$ does admit a nontrivial binary operation, then it is either a topological semilattice or a topological group (by Post's classification, cf. [4]). In both cases $X$ cannot be extremally disconnected [2].

There are also restrictions for second-countable spaces. Recall that $\neg$ denotes the negation. Any subalgebra of $\langle 2 ; \neg\rangle^{I}$ has a continuous involution without fixed point. It follows that its underlying space is of the form $Y \times 2$, which is not true of all second-countable spaces. Another obstacle is the ternary affine sum $s(x, y, z)=x \oplus y \oplus z$ (addition modulo 2). If $X$ admits $s$, then by choosing any element $x_{0}, X$ becomes a group under $x+y=s\left(x, y, x_{0}\right)$. It follows that $X$ must be homogeneous.

We shall see below that $\neg$ and $s$ are, in a sense, the only obstacles for second-countable spaces. Before we can give the exact statement we recall some notation.

By $\bar{F}$ we denote the set of all operations on 2 that can be obtained as superpositions of projections $2^{n} \rightarrow 2$ and operations in $F$. The set $\bar{F}$ is closed under superpositions, i.e., a so-called clone. It is the clone generated by $F$. It is clear that a space admits $F$ iff it admits $\bar{F}$.

All possible clones on 2 are known and are well described [4]. This makes it possible to prove the following result "by inspection", with the hitherto open case of $F=\{m,-\}$ settled above. 
Theorem 3.1. Let $F$ be a set of operations on 2. If $s \in \bar{F}$, then only Cantor cubes admit $F$. If $s \notin \bar{F}$ and $\neg \in \bar{F}$, then the spaces admitting $F$ are those of the form $Y \times \mathbf{2}$. If neither $\neg$ nor $s$ belong to $\bar{F}$, then all second-countable, compact, zero-dimensional spaces admit $F$.

It is an open problem what happens if $\mathbf{2}$ is replaced by a finite set $\mathbf{n}$ with three or more elements. For infinite $I, \mathbf{n}^{I}$ is still homeomorphic to the Cantor cube of weight $|I|$. So every space can be embedded into some $\mathbf{n}^{I}$. We have no nontrivial results concerning embeddings as subalgebras of $\langle\mathbf{n} ; F\rangle^{I}$. Neither is there a classification of clones available, nor do the Boolean algebraic techniques of $\S 1$ work in this case.

\section{REFERENCES}

1. M. G. Bell, The space of complete subgraphs of a graph, Comm. Math. Univ. Carolin. 23 (1983), 525-536.

2. M. G. Bell and J. Pelant, Continuous images of compact semilattices, Canad. Math. Bull. 30 (1987), 109-113.

3. S. Koppelberg, General theory of Boolean algebras, Handbook of Boolean Algebras, Vol. 1 (J. D. Monk and R. Bonnet, eds.), North-Holland, Amsterdam, 1989.

4. E. L. Post, Two-valued iterative systems of mathematical logic, Ann. Math. Studies, vol. 5, Princeton Univ. Press, Princeton, NJ, 1941.

2. Mathematisches Institut, Freie Universität Berlin, Arnimallee 3, D-14195 Berlin, GERMANY

E-mail address: heindorf@math.fu-berlin.de 